\title{
Hepatitis B immunoglobulin in combination with lamivudine for prevention of hepatitis B virus reactivation in children undergoing bone marrow transplantation
}

\begin{abstract}
Tavil B, Kuşkonmaz B, Kasem M, Demir H, Çetin M, Uçkan D. Hepatitis B immunoglobulin in combination with lamivudine for prevention of hepatitis $\mathrm{B}$ virus reactivation in children undergoing bone marrow transplantation.

Pediatr Transplantation 2006: 10:966-969. @ 2006 Blackwell Munksgaard

Abstract: There is little information in literature about the use of hepatitis B immunoglobulin ( $\mathrm{HBIg}$ ) in recipients of bone marrow transplantation (BMT). Here, we report two children who received IV HBIg (Hepatect-CP) and lamivudine treatment during BMT course for either patient or donor hepatitis B virus (HBV) viremia. A four-year-old girl underwent a fully human leukocyte antigen-matched allogeneic BMT for thalassemia major from her mother positive for hepatitis B surface antigen (HBsAg). A 12-yr-old boy with chronic myeloid leukemia, positive for HBsAg and HBV-DNA received a fully HLAmatched allogeneic BMT from his sister in the first chronic phase of the disease. HBIg was successfully used in both cases to prevent HBV reactivation of the recipients. The results of our observations are encouraging and we suggest that HBIg in combination with lamivudine may be used in such cases especially in post-transplant early period to prevent $\mathrm{HBV}$ reactivation.
\end{abstract}

\author{
Betul Tavil', Barıș Kuskonmaz', \\ Muhammed Kasem², Hulya Demir ${ }^{3}$, \\ Mualla Çetin ${ }^{\mathbf{1}}$ and Duygu Uçkan ${ }^{4}$ \\ ${ }^{1}$ Pediatric Hematology Unit, ${ }^{2}$ Ihsan Dogramac1 \\ Children's Hospital, ${ }^{3}$ Pediatric Gastroenterology Unit, \\ ${ }^{4}$ Pediatric Bone Marrow Transplantation Department, \\ Hacettepe University School of Medicine, Sıhhiye \\ Ankara, Turkey
}

Key words: bone marrow transplantation - hepatitis B immunoglobulin - children

Betul Tavil, Pediatric Hematology Unit, Hacettepe University Faculty of Medicine, 06100-S1hhiye Ankara, Turkey

Tel.: 0903122051979

Fax: 0903123243286

E-mail: betultavil@yahoo.com

Accepted for publication 24 May 2006
Viral reactivation is a common problem after allogeneic BMT due to intensive chemotherapy. The risk of HBV reactivation in allogeneic BMT recipients with $\mathrm{HBsAg}$ or transplanted from HBsAg-positive donor has not been clearly defined. It has been reported that positivity for $\mathrm{HBsAg}$ at the time of transplantation is not a contraindication for allogeneic BMT (1). However, HBV reactivation leading to hepatic failure has been reported in allogeneic BMT recipients as well as in other immunocompromised patients. If the recipient or the donor is positive for HBsAg, the patient has been shown to be at greater risk for HBV reactivation and fulminant

Abbreviations: BMT, bone marrow transplantation; CML, chronic myeloid leukemia; GVHD, graft-versus-host-disease; HBIg, hepatitis B immunoglobulin; HBV, hepatitis B virus; HLA, human leukocyte antigen; IV, intravenous; $\mathrm{HBs}$, hepatitis B surface antigen; HBe, hepatitis Be antigen; $\mathrm{HBc}$, hepatitis B core antigen. hepatic failure. In case reports, most of the patients with HBV reactivation had a fulminant course that progressed into hepatic failure and death $(2-4)$.

Lamivudine, a nucleoside analog, has been used to treat HBV infection for many years. Combined lamivudine and IV HBIg treatment has recently been recommended in liver transplantation patients for the prevention of $\mathrm{HBV}$ recurrence after transplantation (5). HBIg and lamivudine treatment may also be useful to prevent severe liver disease in allogeneic BMT recipients.

In this report, we presented two cases of allogeneic BMT recipients; one of the recipient was positive for $\mathrm{HBsAg}$ and the other was transplanted from a donor positive for HBsAg. IV HBIg in combination with lamivudine treatment was successfully used for both cases to prevent HBV reactivation in the recipients. 


\section{Case reports}

Case 1

A four-year-old girl underwent BMT from her HLA-identical mother for class II $\beta$-thalassemia major. She was the product of a first-degree consanguineous marriage and had one brother with thalassemia major. Her mother was an inactive $\mathrm{HBsAg}$ carrier (HBsAg positive, anti$\mathrm{HBs}$ negative, anti-HBc IgM negative, anti-HBc IgG positive, $\mathrm{HBeAg}$ negative, anti-HBe positive, HBV-DNA negative) before BMT. The patient had received HBIg at birth and been vaccinated with four doses of recombinant (at $0,1,2$ and 12 months), yeast derived, HBsAg containing vaccine (Engerix-B; Glaxo SmithKline Biologicals, Rixensart, Belgium). She was anti-HBs positive with low titer (44 IU/L) and $\mathrm{HBsAg}, \mathrm{HBeAg}$, anti-HBe, anti-HBc IgM, anti-HBc IgG, and HBV-DNA were all negative for her. In order to increase the anti-HBs titer, she was given additional dose of hepatitis $\mathrm{B}$ vaccine. Conditioning treatment included cyclophosphamide $(200 \mathrm{mg} /$ $\mathrm{kg})$ and busulfex $(12.8 \mathrm{mg} / \mathrm{kg})$ and GVHD prophylaxis consisted of cyclosporine-A and methotrexate. Because of minor ABO incompatibility, plasma-depleted bone marrow was infused on day 0 . She received HBIg and lamivudine for the prevention of HBV transmission from the donor graft. Therefore, eight doses of 2000 IU HBIg (Hepatect-CP, Biotest Pharma GmbH, Dreieich, Germany) were given at four consecutive days, after then three consecutive weeks, and at fourth month. The initial dose of HBIg was given at day -1 , then during bone marrow infusion, and immediately after the transplantation and continued until four months after BMT. Lamivudine prophylaxis $(4 \mathrm{mg} / \mathrm{kg} /$ day $)$ was also administered from day -1 to +102 . HBIg consumption was assessed by serial measurement of anti-HBs antibody titer. HBIg was readministered, when antiHBs antibody titers dropped below $100 \mathrm{IU} / \mathrm{L}$. She remains in well clinical condition with full donor chimerism (molecular method) at +12 months and $\mathrm{HBV}$ reactivation was not observed during this period. At her last follow-up examination, her anti-HBs antibody titer remained $154 \mathrm{IU} / \mathrm{L}$.

Case 2

A 12-yr-old boy with CML received a fully HLA-matched allogeneic BMT from his sister in the first chronic phase of the disease. He was positive for $\mathrm{HBsAg}, \mathrm{HBeAg}$, and anti-HBc IgG; negative for anti-HBs, anti-HBe, and anti-HBc IgM. His HBV-DNA was also positive with low titer (2828 copies $/ \mathrm{mL}$ ) and the liver function tests were within normal reference range, at the time of BMT. His donor was negative for HBsAg and positive for anti-HBs (83 IU/L). The conditioning regimen consisted of cyclophosphamide $(200 \mathrm{mg} / \mathrm{kg})$ and busulfex $(12.8 \mathrm{mg} / \mathrm{kg})$. Cyclosporine-A and methotrexate were administered for prophylaxis of GVHD. He received HBIg and lamivudine for the prevention of $\mathrm{HBV}$ reactivation. A total of 12 doses of 4000 IU HBIg (Hepatect-CP) was given at six consecutive weeks and then every other week for 12 wk. The initial dose of HBIg was given at day -1 , then during bone marrow infusion, and continued for $18 \mathrm{wk}$ after BMT to maintain anti-HBs level above $100 \mathrm{IU} / \mathrm{L}$. Lamivudine prophylaxis $(4 \mathrm{mg} / \mathrm{kg} /$ day) was also administered starting -1 day of BMT until +100 day. The significant elevation of liver transaminases at day +23 (peak alanine amino transferase/aspartate amino transaminase: 945/1135 IU/L, peak HBVDNA: 89628 copies $/ \mathrm{mL}$ ) was attributed to inflammatory reaction coinciding with the time of engraftment and resolved upon methylprednisolone administration within 10 days. As, the picture resolved upon methylprednisolone administration, liver biopsy was not performed. Methylprednisolone treatment was tapered off within two weeks. He continued to receive HBIg and lamivudine treatment, and he became seropositive for anti-HBs and anti-HBe except for $\mathrm{HBsAg}$ and anti-HBc IgG and his HBV-DNA level was very low (100 copies $/ \mathrm{mL})$. He has been followed-up for 11 months after BMT, and HBV reactivation was not observed in this period. At his last follow-up, he remains clinically well with Karnofsky score $>90 \%$ and full donor chimerism. He did not become $\mathrm{HbsAg}$ negative, whereas he became seropositive for anti-HBs and anti-HBe, which were negative before he received the treatment of $\mathrm{HBIg}$ and lamivudine. The last $\mathrm{HBV}$ serology revealed positive for HBsAg, anti-HBs, anti-HBe, anti-HBc IgG, and HBV-DNA (1740 copies/mL) and negative for $\mathrm{HBeAg}$.

\section{Discussion}

The origin of $\mathrm{HBV}$ infection after BMT includes reactivation of a latent infection or chronic HBV infection, and new HBV infection from BMT donors (6). The risk of $\mathrm{HBV}$ reactivation in patients positive for HBsAg after BMT has been well recognized $(2,7)$. Patients seropositive for anti-HBs from natural infection have also been shown to harbor latent virus, which can also reactivate during the immunocompromised period of BMT (8). 
Studies have shown that positivity for HBsAg at the time of transplantation does not seem to increase the incidence of veno-occlusive disease or GVHD of the liver. In addition, it also does not affect the engraftment and is not associated with graft failure. Thus, HBsAg positivity of patient is not considered as contraindication for BMT (1). However, the possibility of HBV reactivation is a fearful expectation due to fulminant course of the disease.

In these cases, hepatitis may progress to the fulminant stage. It is thought that this was induced by reactivation of $\mathrm{HBV}$ in the recipient's liver cells. The virus begins to proliferate during immunosuppression period and active hepatitis develops depending on the recovery of the immune response after BMT (9). The occurrence of transaminases elevation at day +23 in case 2 was not attributed to hepatitis B reactivation because of low level of the HBVDNA $\left(<10^{5}\right.$ copies $\left./ \mathrm{mL}\right)$. Additionally, transaminase levels returned to normal within 10 days.

Adaptive immunity transfer has been reported to be effective in clearing HBV infection. In a study performed in our center, the changes in HBV serology after BMT has been evaluated in children. Before BMT, 40 children were $\mathrm{HBsAg}$ negative and five were positive. Of these, five children positive for $\mathrm{HBsAg}$, HBsAg disappeared in only two children and anti-HBs became positive in one (10). In an other study performed by Lau et al., among 226 adult patients who received allogeneic BMT, 21 were positive for HBsAg before BMT. Only two of these 21 patients had sustained clearance of HBV infection after BMT (11). As transfer of adaptive immunity is not always possible, we used HBIg and lamivudine treatment in case 2 who was positive for HBsAg.

In an immunized host, anti-HBs alone may not be sufficient to clear the circulating HBV achieved from HbsAg-positive donor and prevent tissue infection (12). As her anti-HBs titer was very low, case 1 (underwent BMT from HBsAg-positive donor) was re-vaccinated before BMT. However, considering the immunosuppressed state of the patient and possible failure of an adequate immune response to clear the virus, HBIg in combination with lamivudine was used and the treatment was effective for protection of the patient from HBV infection.

Lamivudine that inhibits the reverse transcriptase step in HBV replication has been used to prevent $\mathrm{HBV}$ reactivation in immunocompromised recipients for many years. The major limitation to lamivudine is the development of drug resistance, which increase over the duration of therapy $(5,13)$. As the duration of lamivudine therapy should be as short as possible to avoid the development of resistance to the therapy, lamivudine and HBIg combination may be a more useful choice than single agent.

There is little information in the literature about the use of HBIg in recipients of BMT. Irinondo et al. reported a 46-yr-old woman with acute non-lymphocytic leukemia who underwent BMT from her HBsAg-positive HLA-identical brother. They successfully used HBIg and concluded that BMT can be performed from a HBsAg-positive donor if high doses of HBIg are used for prophylaxis against HBV infection (14).

The cost of prophylaxis with HBIg after BMT may be high, whereas if the duration of prophylaxis is limited to the first three months, the most risky period for viral reactivation, with low dosage, it will be highly cost effective. As the consequences of $\mathrm{HBV}$ reactivation is very unfavorable, the use of HBIg may be noteworthy. As the first case was four year old and her body weight was $20 \mathrm{~kg}$, she received eight doses of 2000 IU HBIg. The second case was $12 \mathrm{yr}$ old and his body weight was $40 \mathrm{~kg}$; he received 12 doses of $4000 \mathrm{IU} \mathrm{HBIg}$. The dosage was $1000 \mathrm{IU} \mathrm{HBIg} / 10 \mathrm{~kg}$ and the duration was determined according to the level of anti-HBs antibody titer, which was measured initially at one week, then at two-week intervals after discharge. When anti-HBs antibody titers dropped below $100 \mathrm{IU} / \mathrm{L}$, HBIg was readministered in the first four months. Lamivudine prophylaxis was started -1 day of BMT to approximately +100 day, which is the most risky period for immunosuppression. A fixed schedule cannot be recommended. Because, the clinical and laboratory conditions of the patients were different and the treatment was regulated according to the patient's clinical and laboratory conditions.

To our knowledge, HBIg has not been used previously during BMT course in children positive for $\mathrm{HBsAg}$ or transplanted from HbsAgpositive donor to prevent $\mathrm{HBV}$ reactivation. In the present preliminary study, the number of patients is very limited and further studies are needed to recommend the general use of the treatment. However, the results of our observations are encouraging and we suggest that HBIg and lamivudine combination may be used in such cases especially in the early period of BMT to prevent HBV reactivation. 


\section{References}

1. Reed EC, Myerson D, Corey L, Meyers JD. Allogeneic marrow transplantation in patients positive for hepatitis B surface antigen. Blood 1991: 77: 195-200.

2. Pariente EA, Goudeau A, Dubois F, et al. Fulminant hepatitis due to reactivation of chronic hepatitis $\mathrm{B}$ virus infection after allogeneic bone marrow transplantation. Dig Dis Sci 1988: 33: 1185-1191.

3. Webster A, Brenner MK, Prentice hG, Griffiths PD. Fatal hepatitis B reactivation after autologous bone marrow transplantation. Bone Marrow Transplant 1989: 4: 207-208.

4. Fan FS, Tzeng CH, Yeh HM, Chen PM. Reverse seroconversion of hepatitis B virus infectious status after allogeneic bone marrow transplantation from a carrier donor. Bone Marrow Transplant 1992: 10: 189-191.

5. Dumortier J, Chevallier P, Sconzec JY, Berger F, Boillot O. Combined lamivudine and hepatitis B immunoglobulin for the prevention of hepatitis B recurrence after liver transplantation: long-term results. Am J Transplant 2003: 3: 999-1002.

6. Hashino S, Nozawa A, Izumiyama $\mathrm{K}$, et al. Lamivudine treatment for reverse seroconversion of hepatitis B 4 years after allogeneic bone marrow transplantation. Bone Marrow Transplant 2002: 29: 361-363.

7. Caselitz M, Link H, Hein R, et al. Hepatitis B associated liver failure following bone marrow transplantation. J Hepatol 1997 27: $572-577$.
8. Martin BA, Rowe JM, Kouides PA, Dipersio JF. Hepatitis B reactivation following allogeneic bone marrow transplantation: Case report and review of the literature. Bone Marrow Transplant 1995: 15: 145-148.

9. Inai K, Tashima M, Iтон M, et al. Fulminant hepatitis B following bone marrow transplantation in an HBsAg-negative, $\mathrm{HBs} A$-positive recipient; reactivation of dormant virus during the immunosuppressive period. Bone Marrow Transplant 2000: 25: 105-108.

10. Kupeli S, Ozen H, UcKan D, et al. Changes in hepatitis B virus serology in bone marrow transplanted children. Pediatr Transplant 2002: 6: 406-410.

11. Lau GK, Suri D, Liang R, et al. Resolution of chronic hepatitis B and anti-HBs seroconversion in humans by adoptive transfer of immunity to hepatitis B core antigen. Gastroenterology 2002: 122: 614-624.

12. Chisari FV, Ferrari C. Hepatitis B virus immunopathogenesis. Annu Rev Immunol 1995: 13: 29-60.

13. Doong L, Tsai H, Schinazi F. Inhibition of the replication of hepatitis $\mathrm{B}$ virus in vitro by 2-3-dideoxy-3-thiacytidine and related analogues. Proc Natl Acad Sci USA 1991: 88: 8495 8499.

14. Irinondo A, Pastor JM, Hermosa V, et al. Protection against hepatitis B after marrow transplantation. Ann Intern Med 1986: 105: 293-294. 